\title{
Enumerative Formulae for Unrooted Planar Maps: a Pattern
}

\author{
Valery A. Liskovets* \\ Institute of Mathematics \\ National Academy of Sciences, 220072, Minsk, BELARUS \\ liskov@im. bas-net. by
}

Submitted: Feb 5, 2004; Accepted: Nov 2, 2004; Published: Dec 7, 2004

Mathematics Subject Classifications: 05C30, 05A10

\begin{abstract}
We present uniformly available simple enumerative formulae for unrooted planar $n$-edge maps (counted up to orientation-preserving isomorphism) of numerous classes including arbitrary, loopless, non-separable, eulerian maps and plane trees. All the formulae conform to a certain pattern with respect to the terms of the sum over $t \mid n, t<n$. Namely, these terms, which correspond to non-trivial automorphisms of the maps, prove to be of the form $\phi\left(\frac{n}{t}\right) \alpha r^{t}\left(\begin{array}{c}k t \\ t\end{array}\right)$, where $\phi(m)$ is the Euler function, $k$ and $r$ are integer constants and $\alpha$ is a constant or takes only two rational values. On the contrary, the main, "rooted" summand corresponding to $t=n$ contains an additional factor which is a rational function of $n$. Two simple new enumerative results are deduced for bicolored eulerian maps. A collateral aim is to briefly survey recent and old results of unrooted planar map enumeration.
\end{abstract}

\section{Introduction}

We discover and discuss an unexpected pattern of the formulae for counting non-isomorphic unrooted planar maps derived previously in $[10,12,15,16,17,18]$ : the cancellation of a rational factor in the summands over the divisors $t \mid n, t<n$. Formerly we have not considered these formulae in such an explicit form (rather, we expressed them reductively, that is, in terms of the number of the corresponding rooted maps), nor did we compare them with each other. Two most striking instances of such behaviour have appeared only recently $[17,18]$. The same pattern is also valid for several enumerative formulae derived by other researchers, see $[3,6]$. More than ten results confirming it are considered. Almost all the results are based on the general method of the enumeration of unrooted planar maps developed in $[10,12]$, and two simple new results are deduced in the present paper.

${ }^{*}$ Supported by INTAS and SCST of the Republic of Belarus under grant 03-50-5975.

THE ELECTRONIC JOURNAL OF COMBINATORICS 11 (2004), \#R88 
In this article, a map means a planar map: a proper embedding of a finite connected planar graph (generally with loops and multiple edges) in the (oriented) sphere. A map is called rooted if an edge-end (a vertex-edge incidence pair), called its root, is distinguished in it. The corresponding edge and vertex are said to be rooted. Unlike maps without a distinguished edge-end (called unrooted), rooted maps have only the trivial automorphism.

\subsection{Formulation}

So far, in counting unrooted maps up to orientation-preserving isomorphism we considered mainly classes of planar maps $\mathfrak{M}$ for which the corresponding numbers of rooted $n$-edge maps $M^{\prime}(n)$ are expressed by simple sum-free formulae. In almost all the cases, the latter can be represented in the form

$$
M^{\prime}(n)=\frac{q(n)}{p(n)} r^{n}\left(\begin{array}{c}
k n \\
n
\end{array}\right)
$$

where $k$ and $r$ are some positive integers and $p(n)$ and $q(n)$ are some polynomials with integer coefficients.

Our formulae for the number of unrooted maps $M^{+}(n)$ of the same classes follow a certain pattern. In particular, they contain one summation over the divisors of $n$, which correspond to non-trivial automorphisms of the maps. Moreover, as we will see below (and what is the subject of the present consideration), all these sums can be represented uniformly as follows:

$$
\sum_{t<n, t \mid n} \phi\left(\frac{n}{t}\right) \alpha r^{t}\left(\begin{array}{c}
k t \\
t
\end{array}\right),
$$

where $\phi(m)$ is the Euler totient function, $r$ and $k$ are the same as in (1) and $\alpha$ is a constant or $\alpha=\alpha(t)$ is a simple bounded "rational-factor-free" function of $t$. In other words, in the summands (with $t \neq n$ ), the rational factor $\frac{q(t)}{p(t)}$ disappears completely. In all cases under consideration, $\alpha$ proves to be a constant or, in three related cases, it takes only two rational values.

Sometimes we consider the pattern (2) with respect to maps having $m n$ edges, where $m$ is a constant different from 1 .

The formulae usually contain one or several additional summands depending on the parity of $n$; their presence does not change the pattern.

\subsection{An interpretation}

The enumerative formulae for the majority of classes $\mathfrak{M}$ of unrooted maps considered below have been known to be inexplicably much simpler than we could expect in the framework of the enumerative method used to obtain these formulae [10, 12]: the intermediate sums of the terms corresponding to the classes of quotient maps with respect to rotational automorphisms of order $n / t, t \mid n, t<n$, arising from this method disappear 
ultimately as if these quotient maps formed the same, or almost the same, sets $\mathfrak{M}(t)$. In general, this is not the case: for instance, quotient maps of non-separable maps are not necessarily non-separable, and quotient maps of loopless maps may contain loops. The pattern discussed in the present note, possibly unimportant by itself, manifests a supplementary unexpected regularity: the average number of admissible choices of the rotation axes in the $t$-edge quotient maps so as to lift them back into $\mathfrak{M}(n)$ (the key step of the

enumerative method) coincides with (or is proportional to) the inverse fraction $\frac{p(t)}{q(t)}$. This coincidence is unexplained even for the simple class $\mathfrak{A}(n)$ of arbitrary maps (see Sect. 2.5), where for $t<n / 2$, the quotient maps are also arbitrary $t$-edge maps (rotations of order 2 bring slight complications; they contribute only to one term, which is immaterial in the context of the present paper). What is the nature of this phenomenon, or is it not merely an artefact? This question remains open; hardly an explanation can be obtained in the framework of the considered enumerative method of quotient maps. We will discuss briefly possible generalizations and limitations of the patterns (1) and (2) in the last section.

\section{Counting unrooted maps}

Additional definitions and details concerning the results stated below and their proofs can be found in the corresponding references.

\section{$2.1 \quad$ Loopless maps $\mathfrak{L}$}

According to [30], for rooted loopless maps,

$$
L^{\prime}(n)=\frac{2(4 n+1) !}{(n+1) !(3 n+2) !}=\frac{2(4 n+1)}{(n+1)(3 n+1)(3 n+2)}\left(\begin{array}{c}
4 n \\
n
\end{array}\right) .
$$

Just the inverse fraction $\frac{p(t)}{q(t)}=\frac{(t+1)(3 t+1)(3 t+2)}{2(4 t+1)}$ multiplied by $L^{\prime}(t)$ for $t<n$ appears in the main formula [18, Th.1], which results in the following expression for the number of unrooted loopless maps:

$$
\begin{aligned}
& L^{+}(n)=\frac{1}{2 n}\left[\frac{2(4 n+1)}{(n+1)(3 n+1)(3 n+2)}\left(\begin{array}{c}
4 n \\
n
\end{array}\right)+\sum_{t<n, t \mid n} \phi\left(\frac{n}{t}\right)\left(\begin{array}{c}
4 t \\
t
\end{array}\right)\right. \\
& +\left\{\begin{aligned}
\frac{2 n}{n+1}\left(\begin{array}{c}
2 n \\
\frac{n-1}{2}
\end{array}\right) & \text { if } n \text { is odd } \\
\left(\begin{array}{c}
2 n \\
\frac{n-2}{2}
\end{array}\right) & \text { if } n \text { is even }
\end{aligned}\right] .
\end{aligned}
$$

Thus, we have the pattern (2) with $k=4$ and $\alpha=r=1$. This is the instance with the most non-trivial polynomials $p(n)$ and $q(n)$ (of degrees 3 and 1 respectively). 


\subsection{Non-separable maps $\mathfrak{B}$}

In another terminology, these are 2-connected maps. For these maps by [26],

$$
B^{\prime}(n)=\frac{2(3 n-3) !}{n !(2 n-1) !}=\frac{4}{3(3 n-2)(3 n-1)}\left(\begin{array}{c}
3 n \\
n
\end{array}\right) \text {. }
$$

Thus, $p(n)=3(3 n-2)(3 n-1)$. Now, the sum in the main formula of [15] for the number of unrooted non-separable maps $B^{+}(n)$ contains terms with the factor

$$
1+9\left(\begin{array}{l}
t \\
2
\end{array}\right)=\frac{(3 t-2)(3 t-1)}{2}, \quad t<n
$$

It is just $p(t)$ up to multiplicative constant; therefore this result can be represented in the following form:

$$
\begin{aligned}
B^{+}(n) & =\frac{1}{2 n}\left[\frac{4}{3(3 n-2)(3 n-1)}\left(\begin{array}{c}
3 n \\
n
\end{array}\right)+\frac{2}{3} \sum_{t<n, t \mid n} \phi\left(\frac{n}{t}\right)\left(\begin{array}{c}
3 t \\
t
\end{array}\right)\right] \\
& +\left\{\begin{array}{cc}
\frac{4(n+1)}{3(3 n-1)(3 n+1)}\left(\begin{array}{c}
3 \frac{n+1}{2} \\
\frac{n+1}{2}
\end{array}\right) & \text { if } n \text { is odd } \\
\frac{1}{3(3 n-2)}\left(\begin{array}{c}
3 \frac{n}{2} \\
\frac{n}{2}
\end{array}\right) & \text { if } n \text { is even }
\end{array}\right.
\end{aligned}
$$

Thus, the summands here are of the form (2) with $k=3, r=1$ and $\alpha=2 / 3$.

\subsection{Eulerian maps $\mathfrak{E}$}

These are maps with all vertices of even valency. By [26] and [20] (see also [28]),

$$
E^{\prime}(n)=\frac{3 \cdot 2^{n-1}(2 n) !}{n !(n+2) !}=\frac{3 \cdot 2^{n}}{2(n+1)(n+2)}\left(\begin{array}{c}
2 n \\
n
\end{array}\right) \text {. }
$$

According to [17],

$$
\begin{aligned}
& E^{+}(n)=\frac{1}{2 n}\left[\frac{3 \cdot 2^{n}}{2(n+1)(n+2)}\left(\begin{array}{c}
2 n \\
n
\end{array}\right)+3 \sum_{t<n, t \mid n} \phi\left(\frac{n}{t}\right) 2^{t-2}\left(\begin{array}{c}
2 t \\
t
\end{array}\right)\right] \\
& +\left\{\begin{array}{ll}
\frac{2^{(n-1) / 2}}{n+1}\left(\begin{array}{c}
n-1 \\
\frac{n-1}{2}
\end{array}\right) & \text { if } n \text { is odd } \\
\frac{1}{n} \sum_{t \mid \frac{n}{2}} \phi\left(\frac{n}{t}\right) 2^{t-3}\left(\begin{array}{c}
2 t \\
t
\end{array}\right)+\frac{2^{(n-4) / 2}}{n+2}\left(\begin{array}{c}
n \\
\frac{n}{2}
\end{array}\right) & \text { if } n \text { is even }
\end{array} .\right.
\end{aligned}
$$

Thus, the denominator $(t+1)(t+2)$ has disappeared, and we recognize (taking into account both sums over $t$ ) the pattern (2) with $k=r=2, \alpha=3 / 4$ for odd $n$ and $\alpha=\left\{\begin{array}{cc}1 & \text { if } t \mid \frac{n}{2} \\ 3 / 4 & \text { otherwise }\end{array}\right\}$ for even $n$.

We note that, by duality, $E^{+}(n)$ is also the number of bipartite maps. 


\subsection{Unicursal maps $\mathfrak{U}$}

These are maps with exactly two odd-valent vertices. By [17],

$$
U^{\prime}(n)=2^{n-2}\left(\begin{array}{c}
2 n \\
n
\end{array}\right)
$$

and

$$
U^{+}(n)=\frac{1}{2 n} \sum_{\substack{t \mid n \\
n / t \text { odd }}} \phi\left(\frac{n}{t}\right) 2^{t-2}\left(\begin{array}{c}
2 t \\
t
\end{array}\right)+ \begin{cases}2^{(n-3) / 2}\left(\begin{array}{c}
n-1 \\
\frac{n-1}{2}
\end{array}\right) & \text { if } n \text { is odd } \\
2^{(n-6) / 2}\left(\begin{array}{c}
n \\
\frac{n}{2}
\end{array}\right) & \text { if } n \text { is even }\end{cases}
$$

Thus, with no cancellation (in this degenerate case, $t=n$ is not an exception), this formula meets the pattern (2) with $k=r=2$ and $\alpha=1 / 4$ if $n / t$ is odd and $\alpha=0$ otherwise.

\subsection{Arbitrary maps $\mathfrak{A}$}

According to [26],

$$
A^{\prime}(n)=\frac{2(2 n) ! 3^{n}}{n !(n+2) !}=\frac{2 \cdot 3^{n}}{(n+1)(n+2)}\left(\begin{array}{c}
2 n \\
n
\end{array}\right) .
$$

By Euler's formula, a map with $t$ edges has $t+2$ vertices and faces altogether. Thus we

can choose two axial cells among them in $\left(\begin{array}{c}t+2 \\ 2\end{array}\right)$ ways. This is just the denominator in the right-hand side of formula (12) for $A^{\prime}(t)$. Therefore this fraction disappears in the $t$-summands of the formula for the number of unrooted maps $A^{+}(n)[10,11,12]$ :

$$
\begin{aligned}
A^{+}(n) & =\frac{1}{2 n}\left[\frac{2 \cdot 3^{n}}{(n+1)(n+2)}\left(\begin{array}{c}
2 n \\
n
\end{array}\right)+\sum_{t<n, t \mid n} \phi\left(\frac{n}{t}\right) 3^{t}\left(\begin{array}{c}
2 t \\
t
\end{array}\right)\right] \\
& + \begin{cases}\frac{2 \cdot 3^{(n-1) / 2}}{n+1}\left(\begin{array}{c}
n-1 \\
\frac{n-1}{2}
\end{array}\right) & \text { if } n \text { is odd } \\
\frac{2(n-1) 3^{(n-2) / 2}}{n(n+2)}\left(\begin{array}{c}
n-2 \\
\frac{n-2}{2}
\end{array}\right) & \text { if } n \text { is even }\end{cases}
\end{aligned}
$$

Thus, we get the pattern (2) with $k=2, r=3$ and $\alpha=1$.

\subsection{Plane trees $\mathfrak{T}$}

It is well known (one of diverse interpretations of the Catalan numbers) that

$$
T^{\prime}(n)=\frac{(2 n) !}{n !(n+1) !}=\frac{1}{n+1}\left(\begin{array}{c}
2 n \\
n
\end{array}\right) .
$$


Now, for unrooted plane trees by [27] (cf. also [12]),

$$
\begin{aligned}
T^{+}(n) & =\frac{1}{2 n}\left[\frac{1}{n+1}\left(\begin{array}{c}
2 n \\
n
\end{array}\right)+\sum_{t<n, t \mid n} \phi\left(\frac{n}{t}\right)\left(\begin{array}{c}
2 t \\
t
\end{array}\right)\right. \\
& +\left\{\begin{array}{cc}
\left(\begin{array}{c}
n \\
\frac{n-1}{2}
\end{array}\right) & \text { if } n \text { is odd } \\
0 & \text { if } n \text { is even }
\end{array}\right] .
\end{aligned}
$$

Thus, the terms are of the form (2) with $k=2$ and $\alpha=r=1$.

Formulae (14) and (15) have numerous generalizations. Consider one of them ${ }^{1}$, counting plane $m$-gonal cacti (or Husimi trees), $m \geq 2$. If an $m$-gonal cactus consists of $n$ $m$-gons, then it contains $N=m n$ edges and $v=(m-1) n+1$ vertices; however for $m=2$ we replace all 2-gons (that is, pairs of parallel edges) by single edges and obtain an ordinary plane tree with $N=n$ edges. Now for the number $T_{m}^{\prime}(n)$ of rooted plane $m$-gonal cacti with $n m$-gons we have, according to [3],

$$
T_{m}^{\prime}(n)=\frac{1}{(m-1) n+1}\left(\begin{array}{c}
m n \\
n
\end{array}\right) \text {. }
$$

For unrooted $m$-gonal cacti, formula (88) [3] can be represented as follows:

$$
T_{m}^{+}(n)=\frac{1}{m n}\left[\frac{1}{(m-1) n+1}\left(\begin{array}{c}
m n \\
n
\end{array}\right)+\sum_{t<n, t \mid n} \phi\left(\frac{n}{t}\right)\left(\begin{array}{c}
m t \\
t
\end{array}\right)+\sum_{\substack{s \mid(m, n-1) \\
s>1}} \phi(s)\left(\begin{array}{c}
\frac{m n}{s} \\
\frac{n-1}{s}
\end{array}\right)\right],
$$

where $(m, n-1)$ denotes the greatest common divisor. For a fixed $m$, the last sum is inessential in our context, and we see the behaviour of the form (2) (the first sum) with $k=m$ and $\alpha=r=1$. Formula (14') conforms to (1) as well. This time, however, both patterns are considered with respect to the number of polygonal blocks $n$, which differs (for $m>2$ ) from the number of edges $N$.

Formulae $\left(14^{\prime}\right)$ and $\left(15^{\prime}\right)$ for $m=3$ also counts so-called 2-trees (resp., rooted and unrooted) with $n$ triangles [5].

\subsection{Constellations $\mathfrak{C}_{m}$}

In terms of maps, $m$-constellations (or simply constellations) are maps in which the faces are properly bicolored black and white, all black faces are of size $m$ and the sizes of all white faces are multiples of $m$. All vertices of an $m$-constellation can be (and are considered to be) colored with the colors $1,2, \ldots, m$ in a unique way so that the vertices around each black face are colored with $1,2, \ldots, m$ in counterclockwise order and, moreover, the root vertex is colored with 1 and the other end of the root edge is colored with 2 . An mconstellation with $n$ black faces has $N=m n$ edges. According to [6], the number of such rooted $m$-constellations is

$$
C_{m}^{\prime}(n)=\frac{(m+1) m^{n}}{m((m-1) n+1)((m-1) n+2)}\left(\begin{array}{c}
m n \\
n
\end{array}\right)
$$

\footnotetext{
${ }^{1}$ Our bibliography is incomplete for counting unrooted plane trees and tree-like maps.
} 
and the number of unrooted $m$-constellations with $n$ black faces is

$$
C_{m}^{+}(n)=\frac{1}{m n}\left[\frac{(m+1) m^{n}}{((m-1) n+1)((m-1) n+2)}\left(\begin{array}{c}
m n \\
n
\end{array}\right)+\frac{m+1}{2} \sum_{t<n, t \mid n} \phi\left(\frac{n}{t}\right) m^{t}\left(\begin{array}{c}
m t \\
t
\end{array}\right)\right]
$$

provided that the symmetries have to preserve the colors of the faces and vertices. Now, with respect to the number of black faces, (17) is an instance of the pattern (2) with $r=m$ and $\alpha=\frac{m+1}{2}$.

We note that $m$-constellations for $m=2$ in fact coincide with bipartite maps in which the vertices are properly bicolored. More exactly, any 2-constellation becomes a bipartite map after transforming every black digon into an edge, and vice versa. The point is that the planar bipartite maps are just the maps with faces of even sizes, and like are the white faces of 2-constellations. These arguments explain why formula (16) for $m=2$ coincides with (8). However (17) differs from (9) because of additional restrictions imposed on the symmetries of $m$-constellations. Their difference can be interpreted combinatorially. Specifically,

$$
\widetilde{E}^{+}(n)=2 E^{+}(n)-C_{2}^{+}(n)
$$

enumerates unrooted vertex-bicolored bipartite maps which are self-dual with respect to reversing the colors. Indeed, the vertices of a bipartite map can be colored properly with black and white in two different ways unless it is self-dual in this sense, in which case only one coloring arises up to isomorphism. Equivalently (by vertex-face duality), this is the number of $n$-edge face-bicolored eulerian maps self-dual with respect to reversing the colors.

By comparing formulae (9) and (17) we conclude from (18) that $\widetilde{E}^{+}(n)$ is twice the second term of the right-hand side expression in (9) depending on the parity of $n$. Slightly transforming it we obtain the following.

\section{Proposition 1.}

$$
\widetilde{E}^{+}(n)=\left\{\begin{array}{ll}
\frac{2^{(n+1) / 2}}{n+1}\left(\begin{array}{c}
n-1 \\
\frac{n-1}{2}
\end{array}\right) & \text { if } n \text { is odd } \\
\frac{1}{n}\left[\frac{(3 n+2) \cdot 2^{(n-4) / 2}}{n+2}\left(\begin{array}{c}
n \\
\frac{n}{2}
\end{array}\right)+\sum_{t<\frac{n}{2}, t \mid \frac{n}{2}} \phi\left(\frac{n}{t}\right) 2^{t-2}\left(\begin{array}{c}
2 t \\
t
\end{array}\right)\right] & \text { if } n \text { is even }
\end{array} .\right.
$$

Here are the numerical values of $\widetilde{E}^{+}(n)$ for $n=1,2, \ldots, 14: \quad 1,1,2,4,8,17,40,93$, 224, 538, 1344, 3352, 8448, 21573.

Now restrict ourselves to even $n$. Replacing $n$ with $2 n$, the second row of the equality (19) can be represented as follows:

$$
\widetilde{E}^{+}(2 n)=\frac{1}{2 n}\left[\frac{(3 n+1) \cdot 2^{n}}{4(n+1)}\left(\begin{array}{c}
2 n \\
n
\end{array}\right)+\frac{1}{4} \sum_{t<n, t \mid n} \phi\left(\frac{2 n}{t}\right) 2^{t}\left(\begin{array}{c}
2 t \\
t
\end{array}\right)\right] .
$$

Hence formula (20) conforms to the pattern (2) with $k=r=2$ and $\alpha=\frac{\phi(2 n / t)}{4 \phi(n / t)}$. Now, $\phi(2 k)=\phi(k)$ if $k$ is odd and $\phi(2 k)=2 \phi(k)$ otherwise. Therefore we see that 
$\alpha=\left\{\begin{array}{ll}1 / 4 & \text { if } n / t \text { is odd } \\ 1 / 2 & \text { if } n / t \text { is even }\end{array}\right\}$.

It is interesting to note that unlike other instances of (2), the first term of (20) is not the number of the corresponding rooted maps (with or without bicoloring faces), as the case-by-case analysis of $n=2$ shows $\left(\widetilde{E}^{+}(4)=4\right)$. It would be interesting to find an appropriate combinatorial interpretation of this term.

The enumeration of unrooted $m$-constellations with uncolored vertices is an open problem for $m>2$.

Constellations with a single white face are known as $m$-ary (colored) cacti. They have been counted in [3]; the formulae obtained here for rooted and unrooted cacti are again of the form (1) and (2) respectively.

\subsection{Bi-eulerian maps $\mathfrak{E}_{2}$}

Bi-eulerian maps are maps with both vertices and faces of even valency (size). According to [17], the number of rooted bi-eulerian maps with $2 n$ edges is

$$
E_{2}^{\prime}(2 n)=\frac{2 \cdot 3^{n}}{3(n+1)(2 n+1)}\left(\begin{array}{c}
3 n \\
n
\end{array}\right), \quad n \geq 1
$$

$\left(E_{2}^{\prime}(n)=0\right.$ for odd $\left.n\right)$. It follows that

$$
E_{2}^{\prime}(2 n)=C_{3}^{\prime}(n)
$$

(formula (16)). Moreover, a similar identity holds for unrooted maps of the same types; thus in view of (17) we obtain one more instance of the pattern (2). By duality, any bi-eulerian map admits proper bicolorings both of vertices and faces. There are $4 E_{2}^{\prime}(2 n)$ rooted bi-eulerian doubly bicolored maps with $2 n$ edges. Let $E_{2}^{(+)}(2 n)$ denote the number of such unrooted bi-eulerian bicolored maps considered up to color-preserving isomorphism.

\section{Proposition 2.}

$$
E_{2}^{(+)}(2 n)=C_{3}^{+}(n), \quad n \geq 1
$$

Proof. Any non-trivial color-preserving automorphism of a bi-eulerian doubly bicolored map with $2 n$ edges should be of order $\ell \mid n$ (so that $2 n / \ell$ is even). It is clear that the corresponding quotient map is again a bicolored bi-eulerian map; moreover, the rotation axis cannot intersect any edges. Conversely, any bi-eulerian bicolored map with $2 t=2 n / \ell$ edges is lifted to a bi-eulerian bicolored map with $2 n$ edges with respect to any choice of two axial cells from the vertices and faces. By Euler's formula, there are $\left(\begin{array}{c}2 t+2 \\ 2\end{array}\right)$ possibilities to choose the axial cells. Now applying the general enumerative theorem of $[10,12]$ we obtain immediately

$$
E_{2}^{(+)}(2 n)=\frac{1}{4 n}\left[4 E_{2}^{\prime}(2 n)+\sum_{t<n, t \mid n} \phi\left(\frac{n}{t}\right)\left(\begin{array}{c}
2 t+2 \\
2
\end{array}\right) 4 E_{2}^{\prime}(2 t)\right] .
$$


Substituting the expression of $E_{2}^{\prime}(2 n)$ by (21) into (24) and comparing the result with formula (17) for $m=3$ we obtain, in view of (22), the desired identity (23).

For a possible bijective proof see [23].

Here are the numerical values of $E_{2}^{(+)}(2 n)$ for $n=1,2, \ldots, 9: \quad 1,6,22,174,1479$, 16808, 201834, 2631594, 35965555.

The enumeration of unrooted bi-eulerian maps up to all isomorphisms including colorreversing ones is an open problem.

\subsection{Regular $2 m$-valent maps $\mathfrak{A}_{2 m}$}

Now we touch briefly the case of regular even-valent maps, i.e. maps with all vertices of valency $d=2 m$. Such maps with $n$ vertices contain $N=m n$ edges. According to Tutte [25] in the rooted case,

$$
A_{2 m}^{\prime}(n)=\frac{2}{((m-1) n+1)((m-1) n+2)}\left[\frac{1}{2}\left(\begin{array}{c}
2 m \\
m
\end{array}\right)\right]^{n}\left(\begin{array}{c}
m n \\
n
\end{array}\right) .
$$

Again, like (14') and (16), it is of the form (1) for any $m$ but only with respect to another parameter $n$, in this case, the number of vertices. $A_{2}^{\prime}(n)=A^{\prime}(n)$. We note also that $A_{4}^{+}(n)=A^{+}(n) / 2$ for odd $n$.

In [12] we deduced a formula for $A_{2 m}^{+}(n)$, which is more complicated than (13). In particular it contains a number of summands depending on divisors of $2 \mathrm{~m}$. Only one sum in it is over all divisors $t$ of $n$. It corresponds to rotations about axes through the center-points of two faces. Such rotations generate regular quotient maps with $m t$ edges and, thus, with $(m-1) t+2$ faces. Therefore we can choose two axial faces for liftings in $\left(\begin{array}{c}(m-1) t+2 \\ 2\end{array}\right)$ ways. Comparing this factor with the denominator in (25) for $n=t$ we see that they cancel each other. This means that the formula for $A_{2 m}^{+}(n)$ [12] does follow the pattern (2).

\section{Discussion}

\subsection{Rescaling}

The Catalan-like form (1) when it is applicable for representing the number $M^{\prime}(n)$ looks natural by itself. It has different advantages, for instance, for establishing direct bijective proofs. Such a bijective proof of a formula often makes it possible to reveal another combinatorial interpretation of the rational factor in (1), see, e.g., [8, 21, 22].

However, of course, (1) is not a unique compact and convenient sum-free form: up to alteration of the polynomial factor and divisor in it we could express the right-hand side expression, e.g., in terms of $\left(\begin{array}{c}k n+1 \\ n+1\end{array}\right)$, and so on. But with respect to such shifted or modified parameters, a superfluous polynomial factor or/and divisor would appear in the summands in (2). Just the absence of a rational factor in formula (2) distinguishes 
expression (1) (one and the same in all the cases, with its "integer-fold" binomial $\left(\begin{array}{c}k n \\ n\end{array}\right)$ !) among other equivalent representations.

Our method [10, 12] for counting unrooted planar maps easily explains a distinction between the term corresponding to $t=n$ and the other terms: unlike the former, which corresponds to the trivial automorphism, the latter contain a factor expressing possible choices of rotational axes (pairs of axial elements) admissible for lifting. But these reasons do not explain the particular phenomenon discussed above and a special preferable role of the form (1).

\subsection{Limitations}

It would be interesting to find new samples of the pattern. However, we cannot expect a lot of them, at least with respect to the number of edges. The point is that the pattern (2) depends heavily on the rooted pattern (1), but only few classes of maps are known to satisfy the latter. Moreover, there are several constraints imposed naturally on the eligibility of formula (1) with respect to the number of edges. First of all, functions in its right-hand side are restricted to those that give rise to an integer for all admissible $n$ (see below). Besides, typically the degree of the denominator $p(n)$ must exceed that of the numerator $q(n)$ by 2 in order to ensure the "standard" critical exponent $-5 / 2$ in the asymptotics $M^{\prime}(n) \sim \gamma n^{-5 / 2} \beta^{n}$ as $n \rightarrow \infty$ (this exponent is to be modified appropriately for maps having special faces or vertices; such are, e.g., unicursal maps and outerplanar maps including trees). Now, in this asymptotic formula for $\mathfrak{A}$ (Sect. 2.5), $\beta=12$ by (12) and Stirling's formula. This implies that for any subclass of maps $\mathfrak{M} \subseteq \mathfrak{A}$, the integers $r$ and $k$ in (1) must satisfy the inequality $r k^{k} /(k-1)^{k-1} \leq 12$, whence $k \leq 4, r \leq 3$ for $k=2$ and $r=1$ for $k=3,4$. Of course, these upper bounds are not valid for wider classes of maps such as colored maps.

\subsection{Promising classes of maps}

There are several classes of maps which confirm the pattern (1) and which we consider to be most interesting for study with respect to the pattern (2).

The first class to be mentioned is the class $\mathfrak{B}_{3}$ of non-separable trivalent maps with $N=3 n$ edges. By [19],

$$
B_{3}^{\prime}(3 n)=\frac{2^{n}(3 n) !}{(2 n+1) !(n+1) !}=\frac{2^{n}}{(n+1)(2 n+1)}\left(\begin{array}{c}
3 n \\
n
\end{array}\right) .
$$

Finding $B_{3}^{+}(3 n)$ still remains an open problem [16].

We return to $L^{\prime}(n)$ (formula (3)). It is well-known that $L^{\prime}(n)$ is also equal to the number of rooted 3-connected planar triangulations with $3 n+3$ edges [24]. Unfortunately, such an equality does not hold for unrooted maps, and no enumerative results of the same nature as are considered in the present paper have been obtained until now for unrooted 3-connected (polyhedral) maps, see [16]. 
Likewise, $E^{\prime}(n)$ (formula (8)) is also equal to the number of rooted bipartite trivalent (bicubic) maps with $3 n$ edges [26]. Enumeratively, rooted bicubic maps proved to be related to diverse types of combinatorial objects, cf. [1, 7]. A simple bijection between rooted bicubic and rooted eulerian maps was established in [20]. However, no formula is known for counting bicubic maps as unrooted ones. They are not equinumerous to unrooted eulerian maps. For example, there are two eulerian maps with 2 edges: a double loop (admits two rootings) and a digon (one rooting), but only one bicubic planar map with 6 edges: a quadrangle in which two opposite edges are doubled (three rootings).

As a counter-example let us consider the class of all trivalent maps $\mathfrak{A}_{3}$. Such maps may contain multiple edges and loops. There is a sum-free formula for the number of rooted trivalent maps with $3 n$ edges obtained by Mullin [20]:

$$
A_{3}^{\prime}(3 n)=3 n(3 n-2) \cdots(n+6)(n+4) \cdot 2^{2 n+1} /(n+1) !, \quad n \geq 2 .
$$

However it can be represented in the appropriate binomial form only for even $n=2 s$ : $A_{3}^{\prime}(6 s)=\frac{2^{6 s}}{(2 s+1)(s+1)}\left(\begin{array}{c}3 s \\ s\end{array}\right)$. A formula for unrooted trivalent maps was obtained in [16]. It resembles that for $A_{2 m}^{+}(n)$ (see Sect. 2.9) and contains a sum over divisors of $n$ including the odd ones. Therefore neither the whole formula nor its restriction to even $n$ conforms to (2) (this does not prove, however, that a (2)-like formula is impossible at all).

Can the pattern (2) be generalized fruitfully to maps with less elementary rooted enumerators? In particular, the following classes of maps could be analyzed in this respect: maps with two faces [4] and maps specified by the number of edges and vertices [29]. It would be interesting to consider some multiparametric classes of maps for which the rooted enumerators are known and are sum-free such as maps of several types with a given vertex valency distribution: eulerian, cacti and other.

\subsection{Polynomial divisors of $\left(\begin{array}{c}k n \\ n\end{array}\right)$}

As a matter of fact, the binomial coefficients $\left(\begin{array}{c}k n \\ n\end{array}\right)$ have a lot of polynomial divisors (up to multiplicative constant). Answering my question, J. Germain obtained the complete description of them as follows:

Proposition 3 [9]. Let $p=p(x)$ be a polynomial with integer coefficients. Then $p(n)$ divides $\left(\begin{array}{c}k n \\ n\end{array}\right), k>1$, for all natural $n$ up to a multiplicative constant $\alpha=\alpha_{p}$ if and only if $p(x)$ is a multiple of the product of a finite set of distinct linear factors $(a x+c)$ and $(b x-d)$ with pairwise coprime natural coefficients, where $a \mid(k-1)$ and $b \mid k, b>1$.

By now I know only one family of maps for which the rooted enumerators are of the form (1) with the denominators of arbitrary degrees. Namely, the formula from [17] for the number $U^{\prime}\left(2 d_{1}+1,2 d_{2}+1 ; n\right)$ of rooted unicursal maps with $n$ edges and two vertices of the odd valencies $2 d_{1}+1$ and $2 d_{2}+1$ can be rewritten in the following form:

$$
U^{\prime}\left(2 d_{1}+1,2 d_{2}+1 ; n\right)=\frac{q_{d}(n) \cdot 2^{n}}{(2 n-1)(2 n-3) \cdots(2 n-2 d-1)}\left(\begin{array}{c}
2 n \\
n
\end{array}\right),
$$


where $d:=d_{1}+d_{2}, d \geq 0$ and $q_{d}(n)$ is a certain polynomial of degree $d+1$ (in general, however, we do not know whether this fraction is uncancellable).

We also note that enumerators of the form $\left(\begin{array}{c}k n \\ n\end{array}\right) / p(n)$ with various polynomials $p(n)$ appeared in the literature in another context related to ours but different from it, see [2].

\subsection{The last term}

Almost always, the last additional summand in the formulae for unrooted maps corresponds to the rotations of order 2 around the midpoints of one or two edges. In all the cases where it arises, it is in fact sum-free and of a form like (1) as well and usually has different values for odd and even $n$. But we do not see an instructive regularity in the behaviour of its rational factor.

\subsection{Summation}

Is it possible, at least in some cases, to simplify (convert) sums (2) so as to get rid of summation with an unrestricted number of terms?

The initial numerical values of some relevant sums (2) are the following:

$$
\begin{aligned}
& \left\{\frac{1}{2} \sum_{t \mid n} \phi\left(\frac{n}{t}\right)\left(\begin{array}{c}
2 t \\
t
\end{array}\right)\right\}_{n=1,2, \ldots}=1,4,12,40,130,480,1722,6480,24336, \ldots \\
& \left\{\frac{1}{4} \sum_{t \mid n} \phi\left(\frac{n}{t}\right) 2^{t}\left(\begin{array}{c}
2 t \\
t
\end{array}\right)\right\}_{n=1,2, \ldots}=1,7,42,288,2020,14838,109830,823976,6223446, \ldots \\
& \left\{\frac{1}{6} \sum_{t \mid n} \phi\left(\frac{n}{t}\right) 3^{t}\left(\begin{array}{c}
2 t \\
t
\end{array}\right)\right\}_{n=1,2, \ldots}=1,10,92,956,10210,112376,1250970,14074312, \ldots \\
& \left\{\frac{1}{3} \sum_{t \mid n} \phi\left(\frac{n}{t}\right)\left(\begin{array}{c}
3 t \\
t
\end{array}\right)\right\}_{n=1,2, \ldots}=1,6,30,172,1005,6228,38766,245336,1562337, \ldots \\
& \left\{\frac{1}{4} \sum_{t \mid n} \phi\left(\frac{n}{t}\right)\left(\begin{array}{c}
4 t \\
t
\end{array}\right)\right\}_{n=1,2, \ldots}=1,8,57,464,3880,33720,296016,2630048, \ldots
\end{aligned}
$$

\subsection{Different approaches to counting unrooted planar maps}

$\mathrm{N}$. Wormald has developed an alternative general method of counting unrooted planar maps $[31,32]$. This method makes it possible to enumerate maps up to sense-reversing homeomorphism as well. But it does not seem to result in closed formulae like the ones considered above for maps on the oriented sphere even in the case of arbitrary maps (cf. formula (13)). Another technique for counting planar maps up to sense-reversing homeomorphism which generalizes the method discussed in this paper has been developed by the author in [13], see also $[14$, Sect.7,8]. 


\section{Acknowledgement}

I am thankful to Timothy Walsh for valuable comments and suggested corrections.

\section{References}

[1] Bóna, M., Exact enumeration of 1342-avoiding permutations: a close link with labeled trees and planar maps. J. Combin. Theory Ser. A 80:2 (1997), 257-272.

[2] Bóna, M. A survey of stack-sorting disciplines, Electron. J. Combin. 9:2 (2003), \#A1, 16 p.

[3] Bóna, M., Bousquet, M., Labelle, G., and Leroux, P. Enumeration of $m$-ary cacti, Adv. in Appl. Math. 24:1 (2000), 22-56.

[4] Bousquet, M., Labelle, G., and Leroux, P. Enumeration of planar two-face maps, Discr. Math. 222 (2000), 1-25.

[5] Bousquet, M., and Lamathe, C. Enumeration of solid 2-trees according to edge number and edge degree distribution, Preprint, submitted to Discr. Math. (see also Proc. 14th Intern. Conf. Formal Power Series and Algebr. Combin. (FPSAC'02), Univ. of Melbourne, Australia, 2002, 133-147).

[6] Bousquet-Mélou, M., and Schaeffer, G. Enumeration of planar constellations, Adv. in Appl. Math. 24:4 (2000), 337-368.

[7] Cori, R., and Schaeffer, G. Description trees and Tutte formulas. Theoret. Comput. Sci. 292:1 (2003), 165-183.

[8] Del Lungo, A., Del Ristoro, F., and Penaud, J.-G. Left ternary trees and nonseparable rooted planar maps, Theoret. Comput. Sci. 233 (2000), 201-215.

[9] Germain, J. Polynomial divisors of binomial(kn,n), unpublished (see letter \#2323 of 18 October 2003 in the Number Theory List http://listserv.nodak.edu/archives/nmbrthry.html).

[10] Liskovets, V.A. A census of non-isomorphic planar maps, In: Algebraic methods in graph theory (Szeged, 1978), Vol. II, 479-494, Colloq. Math. Soc. János Bolyai, 25, North-Holland, Amsterdam, 1981.

[11] Liskovets, V.A. Enumeration of non-isomorphic planar maps, J. Graph Th. 5:1 (1981), 115-117.

[12] Liskovets, V.A. Enumeration of nonisomorphic planar maps, Selecta Math. Soviet. 4:4 (1985), 303-323 (Transl. from: Enumeration of nonisomorphic planar maps (Russian). I, Problems in group theory and homological algebra, 103-115, Yaroslav. Gos. Univ., 1981; II, Geometric methods in problems in analysis and algebra, 106117, 129, 132, Yaroslav. Gos. Univ., Yaroslavl', 1981).

[13] Liskovets, V. A. A reductive technique for enumerating non-isomorphic planar maps. Discr. Math. 156 (1996), 197-217. 
[14] Liskovets, V. Reductive enumeration under mutually orthogonal group actions, Acta Appl. Math. 52 (1998), 91-120.

[15] Liskovets, V.A., and Walsh, T.R.S. The enumeration of non-isomorphic 2connected planar maps, Canad. J. Math. 35:3 (1983), 417-435.

[16] Liskovets, V.A., and Walsh, T.R.S. Ten steps to counting planar graphs, Congr. Numer. 60 (1987), 269-277.

[17] Liskovets, V.A., and Walsh, T.R.S. Enumeration of eulerian and unicursal planar maps, Discr. Math. 282 (2004), 209-221.

[18] Liskovets, V.A., and Walsh, T. R. S. Counting unrooted loopless planar maps, Eur. J. Combin., in press.

[19] Mullin, R. C. On counting rooted triangular maps, Canad. J. Math. 17:3 (1965), 373-382.

[20] Mullin, R. C. On the average number of trees in certain maps, Canad. J. Math. 18:1 (1966), 33-41.

[21] Poulalhon, D., and Schaeffer, G. A bijection for triangulations of a polygon with interior points and multiple edges, Theoret. Comput. Sci. 307 (2003), 385-401.

[22] Schaeffer, G. A combinatorial interpretation of super-Catalan numbers of order two, Manuscript, 2001, 2 p. (available at http://www.lix.polytechnique.fr/〜schaeffe/Biblio/Sc03.ps.gz).

[23] Szabo, R. J., and Wheater, J. F. Curvature matrix models for dynamical triangulations and the Itzykson - Di Francesco formula, Nucl. Phys. B 491 (1997), 689-723 (also arXiv preprint hep-th/9609237).

[24] Tutte, W. T. A census of planar triangulations, Canad. J. Math. 14:1 (1962), 21-38.

[25] Tutte, W. T. A census of slicings, Canad. J. Math. 14:4 (1962), 708-722.

[26] Tutte, W. T. A census of planar maps, Canad. J. Math. 15:2 (1963), 249-271.

[27] Walkup, W. The number of planar trees, Mathematika 19:2 (1972), 200-204.

[28] Walsh, T.R.S. Hypermaps versus bipartite maps, J. Combin. Theory Ser. B 18:2 (1975), 155-163.

[29] Walsh, T.R. S. Efficient enumeration of sensed maps, Discr. Math., in press.

[30] Walsh, T.R.S., and Lehman, A.B. Counting rooted maps by genus. III: Nonseparable maps, J. Combin. Theory Ser. B 18:3 (1975), 222-259.

[31] Wormald, N. C. On the number of planar maps, Canad. J. Math. 33:1 (1981), 1-11.

[32] Wormald, N. C. Counting unrooted planar maps, Discr. Math. 36:2 (1981), 205225. 